Annals of Plant Sciences

ISSN: 2287-688X

OPEN ACCESS

Research Article

www.annalsofplantsciences.com

\title{
Additions to the flora of Srikakulam district, Andhra Pradesh, India
}

Pragada Venkata Ramana

Government Junior College, Kaviti, Srikakulam District, Andhra Pradesh-532322, India.

Received: 2017-10-28; Accepted: 2017-11-03

\begin{abstract}
While exploring the floristic wealth of Srikakulam district of Andhra Pradesh, 43 species of angiospermshas not been reported from Srikakulam district, hence here reported as a new addition to the flora of
\end{abstract} Srikakulam district of Andhra Pradesh.

Keywords: additions; Andhra Pradesh, Eastern Ghats, flora, Srikakulam District

\section{Introduction}

Documentation of the species is very important aspect in the field of taxonomy as well as for further scientific research. In the early fifties, Botanical Survey of India (BSI) was undertaken floristic study of the forests in different districts of the region. R.S. Rao \& Hara Sreeramulu (1986) studied the flora of Srikakulam district and enumerated 916 species of Angiosperms belonging to 417 genera and 114 families. Rama Rao \& Ravi Shankar (1988) reported 38 species as new records of plants from Srikakulam district. Swain et al., (2008) worked on the mangrove habitats of east coast of Srikakulam district and reported ten species including four mangrove species as additions to the flora Srikakulam district. In addition to above taxonomic works, Prayaga Murthy (2009) worked on the weed flora of Uttara Andhra and reported 532 species belonging to 308 genera and 80 families. While exploring the floristic wealth of Srikakulam district, the author has collected and identified 43 species of angiosperms. Scrutiny of literatures revealed that 43 species has not been reported from Srikakulam district (R.S. Rao \& Hara Sreeramulu, 1986; Rama Rao \& Ravi Shankar, 1988; Swain et al., 2008; Prayaga Murthy, 2009), hence here reported as new distributional records for the district of Srikakulam.

\section{Study area}

The Srikakulam district is situated in the northern part of Andhra Pradesh and lies between $18^{\circ} 5^{\prime}$ $19^{\circ}-12^{\prime} \mathrm{N}$ and $83^{\circ} 32^{\prime}-84^{\circ} 47^{\prime} \mathrm{E}$ and bounded by Odisha on the north, Vizianagaram district on the west and south, Bay of Bengal on the east. It occupies an area of $5,837 \mathrm{~km}^{2}$ (2,254 sq mi). The Eastern Ghats run from the northeast of the district of this one of the highest peaks Mahendragiri also partly occurred on this district. The Srikakulam district is one of the biodiversity rich areas of Eastern Ghats. The hilly regions in

\section{*Corresponding Author:}

Dr. Pragada Venkata Ramana,

Government Junior College,

Kaviti, Srikakulam District,

Andhra Pradesh-532322, India.

E-mail: pragadavr@gmail.com
Srikakulam district consists of parts of Palakonda, Pathapatnam, Mandasa and Sompeta which lie in north-west and northern part of the district.

\section{Materials and Methods}

The exploration of the study area was carried out during the period from 2008 to 2016. The plant specimens were collected either in flowering and/or fruiting stage. Detailed study of the dried specimens and their identification were carried in the Department of Botany, Government Junior College, Kaviti, Srikakulam district, with the help of region flora such as Flora of the Presidency of Madras (Gamble, 1915-1936), and other recent floristic works such as those of Pullaiah\&Chennaiah (1997), Pullaiah \& Ali Moulali (1997) and Pullaiah (1997), as well as local district flora by R.S. Rao and Hara Sreeramulu (1986) were consulted. For the processing of herbarium specimens, the methodology described by Jain \& Rao (1977) has been followed. The identified specimens were further confirmed by experts at Deccan Regional Centre (BSID), Hyderabad. All the specimens were deposited in the Department of Botany, Andhra University Visakhapatnam and few specimens at BSID, Hyderabad. The systematic enumerations of all families were arranged alphabetically. Each species is provided with correct name, followed by name of the family, short description, phenology, locality and field numbers.

\section{Results and Discussion}

The present floristic investigation reveals that a total of the 43-plant species belonging to 41 genera and 23 families were identified as new addition to the flora of Srikakulam district. Of these 43 species, 38 species belonging to 36 genera and 19 families are dicotyledonous whereas 5 species belonging to 5 genera and 4 families are monocotyledonous. On

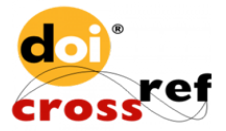


analysis of the habit forms it is found that herbs dominate with 29 herbs, 6 shrubs, 6 climbers and 2 trees.

\section{Enumaration}

Acalypha lanceolata Willd. - EUPHORBIACEAE

Annual erect herb. Leaf ovate - lanceolate, copper green. Flowers in axillary spikes, male flowers few clustered at top, female flowers many, bracts cup shape and teethed.

Fl. \& Fr.: June - August

Specimens examined: Andhra Pradesh, Srikakulam, Kaviti, Ramayyaputtuga, 20 ${ }^{\text {th }}$ June 2016, PV Ramana 22260 (AUV).

Abutilon crispum (L.) Medicus - MALVACEAE

Annual erect herb. Leaves cordate. Flowers axillary, solitary, light yellow. Fruit schizocarp.

Fl. \& Fr.: September- February

Specimens examined: Andhra Pradesh, Srikakulam, Kaviti, Ramayyaputtuga, 5 ${ }^{\text {th }}$ January 2017, PV Ramana 22253 (AUV).

\section{Annona muricata L. - ANNONACEAE}

Small trees. Leaves ovate-lanceolate. Flowers pale yellow. Fruits sub-globose, green, covered with fleshy thick spines.

Fl: August - October Frt.: November - April Specimens examined: Andhra Pradesh, Srikakulam, Kaviti, Jagathi $17^{\text {th }}$ October 2009, PV Ramana 22232 (AUV).

Aponogeton echinatus Roxb. APONOGETONACEAE

Perennial, submerged or floating aquatic herbs. Submerged leaves oblong-elliptic, floating ones oblong. Flowers whitish-pink.

Fl. \& Frt.: December - April

Specimens examined: Andhra Pradesh, Srikakulam,

Kaviti, Kottha Kojjaria $8^{\text {th }}$ May 2013, PV Ramana 22244 (AUV).

Argyreia pilosa Wight \& Arn.-

CONVOLVULACEAE

A large hirsute twiner. Leaves deltoid ovate. Flowers blue in axillary corymbose cymes. Berries yellowish-red when ripe.

Fl. \& Frt.: November, December

Specimens examined: Andhra Pradesh, Srikakulam,

Kaviti, Sivaalayam, $16^{\text {th }}$ December 2008, PV

Ramana 22206 (AUV).

Adenostemma lavenia(L.) Kuntze - ASTERACEAE

Annual erect herb. Leaves ovate to ovate-laceolate. Heads homogamous in terminal corymbs. Flowers white. Achenes obovate, 3-ribbed, and sticky.

Fl. \& Frt.: October - January

Specimens examined: Andhra Pradesh, Srikakulam,

Kaviti, Putiyadala Paddy fields, $21^{\text {st }}$ December 2008 PV Ramana 22207 (AUV).
Alternanthera ficoidea (L.) Sm.- AMARANTHACEAE Prostrate or erect, hairy, branched, annual herb. Leaves narrowly elliptic. Flowers whitish in axillary short globose heads.

Fl. \& Frt.: August-November

Specimens examined: Andhra Pradesh, Srikakulam,

Kaviti, Rajapuram substation, $17^{\text {th }}$ November 2016 , PV Ramana 22263 (AUV).

Alternanthera paronychioides A. St-Hil. -

AMARANTHA CAE

Much branched prostrate creeping hairy herbs. Leaves spathulate or oblanceolate, clustered at nodes. Flowers pale yellow, in axillary and terminal globose heads.

Fl. \& Frt.: January-July

Specimens examined: Andhra Pradesh, Srikakulam, Kaviti, Putiyadala paddy fields, 7th $^{\text {th }}$ ay 2016, PV Ramana 22259 (AUV).

Bergia ammannioides Roxb. ex RothELATINACEAE

Prostrate, wiry, annual herbs. Leaves linear obovate or linear lanceolate. Flowers red in axillary fascicles. Seeds slightly curved.

Fl. \& Frt.: December-April

Specimens examined: Andhra Pradesh, Srikakulam, Kaviti, Putiyadala paddy fields, $8^{\text {th }}$ May 2015, PV Ramana 22252 (AUV).

Capparis brevispina DC. - CAPPARACEAE

Shrubs. Leaves elliptic to oblong. Flowers solitary, axillary, petals white, or pale creamy, upper petals with yellow blotch turned reddish brown later. Stamens 24-40. Berry ellipsoid, red when ripe.

Fl. \& Frt.: February- August

Specimens examined: Andhra Pradesh, Srikakulam, Kaviti, Kapasukddi, Mutyalapeta, $8^{\text {th }}$ May 2013, PV Ramana 22245 (AUV).

\section{Combretum albidum G.Don - COMBRETACEAE}

Large climbing shrubs. Leaves ovate, ovate-elliptic. Flowers pale white, in axillary or terminal long panicles of spikes.

Fl. \& Frt.: Feb-May

Specimens examined: Andhra Pradesh, Srikakulam, Kaviti, Jagathi, 8th May 2013, PV Ramana 22246 (AUV).

Desmodium heterophyllum (Willd.) DC. -

LEGUMINOSAE

Procumbent, branched, hairy herbs. Leaves 3foliate. Flowers white or pinkish white, in leafopposed racemes. Pods oblong, upper suture straight.

Fl. \& Frt.: December to May

Specimens examined: Andhra Pradesh, Srikakulam,

Kaviti, Putiyadala paddy fields, $18^{\text {th }}$ March 2016, PV

Ramana 22253 (AUV). 
Diplocyclos palmatus (L.) C.Jeffery -

CUCURBITACEAE

Annual, slender, tendril climber. Leaves palmately 5-lobed. Flowers monoecious, yellow, in axillary clusters. Fruit spherical, brick red when ripe with white streaks.

Fl. \& Frt.: April-December

Specimens examined: Andhra Pradesh, Srikakulam,

Kaviti, College ground, $8^{\text {th }}$ May 2013, PV Ramana 22246 (AUV).

\section{Dolichos trilobus L. - LEGUMINOSAE}

Tender twiners. Leaves 3-foliate. Flowers pinkish violet or light yellow, in slender axillary racemes. Pods linear.

Fl. \&Frt.: August-February

Specimens examined: Andhra Pradesh, Srikakulam, Kaviti, Ramayyaputtuga, $5^{\text {th }}$ October 2015, PV Ramana 22230 (AUV).

\section{Dyschoriste vagans (Wight) Kuntze -}

\section{ACANTHACEAE}

Erect or sub-erect herbs. Leaves lanceolate, long petiole. Flowers pale white, in axillary short cymes. Fl. \& Frt.: November-December

Specimens examined: Andhra Pradesh, Srikakulam, Kaviti, Rajapurammetta, 16 ${ }^{\text {th }}$ December 2016, PV Ramana 22264 (AUV).

Eleiotis monophylla (Burm.f.) DC. -

LEGUMINOSAE

Annual prostrate herb, branches arising from base, $30 \mathrm{~cm}$ to more than $1 \mathrm{~m}$ high, branchlets triquetrous. Leaves 1-3 foliate, leaflets suborbicular. Flowers pink in lax racemes. Pods boat shaped.

Fr. \&Frt: Most part of the year

Specimens examined: Andhra Pradesh, Srikakulam,

Kaviti, Kapasukiddi, Mutyalapeta, 9 ${ }^{\text {th }}$ June 2012, PV Ramana 22259 (AUV).

Epaltes divaricata (L.) Cass - ASTERACEAE

Erect annual herbs, branches winged. Leaves linear oblong to sub-spathulate. Heads solitary, terminal, pink heterogamous.

Fl. \& Frt.: December-March.

Specimens examined: Andhra Pradesh, Srikakulam, Kaviti, Kottapalem, fields near to coast $26^{\text {th Jamiary }}$ 2015, PV Ramana 22248 (AUV).

Hygrophila ringens (L.) R.Br. ex Steud. ACANTHACEAE

Erect branched herb. Rooting at lower nodes. Leaves ovate, lanceolate or elliptical. Flowers purple-blue, in axillary whorls. Capsule oblong. Fl. \& Frt.: November-March

Specimens examined: Andhra Pradesh, Srikakulam, Kaviti, Putiyadala paddy fields, $18^{\text {th }}$ November 2012, PV Ramana 22242 (AUV).
Indigofera glabra L. - LEGUMINOSAE

Annual diffuse herb. Leaves 3-7 pinnate, leaflets obovate or elliptic. Flower reddish-pink, in axillary racemes. Pods straight.

Fl. \& Frt.: July-October

Specimens examined: Andhra Pradesh, Srikakulam, Kaviti, Kapasukuddi, Mutyalapeta, 23 ${ }^{\text {th }}$ September 2009, PV Ramana 22215 (AUV).

Indigofera wightii Wight \&Arn. - LEGUMINOSAE

Erect under shrubs. Leaves odd pinnate, leaflets elliptic. Flowers pinkish-red, in axillary racemes. Pods straight.

Fl. \& Frt.: September-February

Specimens examined: Andhra Pradesh, Srikakulam, Kaviti, Pragadaputtuga, 30 ${ }^{\text {th }}$ August 2015, PV

Ramana 22251 (AUV).

Lemna gibba L. - ARACEAE

A small, green, floating, stemless, solitary herbs. Fronds floating, suborbicular or obovate.

Fl. \& Frt.: October-December

Specimens examined: Andhra Pradesh, Srikakulam,

Kaviti, Pukkallapalem, $4^{\text {th }}$ October 2009, PV

Ramana 22216 (AUV).

Lepisanthes rubiginosa (Roxb.) Leenh. -

SAPINDACEAE

Medium sized trees, stems and branches clothed with dense rusty-golden tomentum. Leaves odd pinnate. Flowers greenish-white, polygamodioceious, in elongated erect terminal panicles.

Fl. \& Frt.: March-May

Specimens examined: Andhra Pradesh, Srikakulam,

Kaviti to Jagathi roadside $21^{\text {th }}$ April 2011, PV

Ramana 22239 (AUV).

Macrotyloma ciliatum (Willd.) Vetdc. -

LEGUMINOSAE

A twinning silky hairy herbs. Leavs 3-foliolate. Flowers yellow with pinkish-blue, in axillary 3-7 flowered corymbose racemes with 1-7 nodes. Fruit compressed.

Fl. \& Frt.: November-March

Specimens examined: Andhra Pradesh, Srikakulam, Kaviti, Kapasukuddi, 22th December 2014, PV

Ramana 22250 (AUV).

\section{Malachra capitata (L.) L. - MALVACEAE}

Herbs or under shrubs; branches coarsely hairy. Leaves orbicular or palmately 3-5 lobed. Flowers yellow.

Flr\& Frt.: August - October Specimens examined: Andhra Pradesh, Srikakulam, Kaviti, N.H-16 near KotthaKojjaria 19 ${ }^{\text {th }}$ October 2009, PV Ramana 22233 (AUV). 
Mallotus repandus (Willd.) Mull.Arg. -

EUPHORBIACEAE

Large climbing shrub, dioecious. Leaves broadly deltoid-ovate or rhomboid-ovate, very slightly peltate. Flowers in panicled racemes.

Fl. \& Frt.: December-February

Specimens examined: Andhra Pradesh, Srikakulam, Kaviti, Putiyadala, 23 ${ }^{\text {rd }}$ April 2011, PV Ramana 22239 (AUV).

Mikania micrantha (L.) Kunthex H.B.K ASTERACEAE

Much branched climber. Leaves cordate, palmately 5 nerved. Inflorescence in axillary or terminal compound umbels, heads homogamous, white.

Fl. \& Frt.: December - April

Specimens examined: Andhra Pradesh, Srikakulam, Kaviti, Kottapalem 7th December 2014, PV Ramana 22249 (AUV).

Monochoria vaginalis (Burm.f.) C.Presl -

PONTEDERIACEAE

A rooted aquatic herb. Leaves very variable, linear to ovate or ovate-cordate. Flowers blue.

Fl. \& Frt.: August to April

Specimens examined: Andhra Pradesh, Srikakulam,

Kaviti, Putiyadala, ${ }^{\text {st }}$ September 2009, PV Ramana 22215 (AUV).

\section{Nesaea brevipes Koehne - LYTHRACEAE}

Herbs up to $70 \mathrm{~cm}$. Leaves sessile, oblong or elliptic. Flowers pinkish-red, in axillary dichasial cymes. Capsule globose.

Fl. \& Frt.: December-April

Specimens examined: Andhra Pradesh, Srikakulam,

Kaviti, Putiyadala paddy fields, $16^{\text {th }}$ January 2009, PV Ramana 22208 (AUV).

Ottelia alismoides (L.) Pers. HYDROCHARITACEAE

Submerged aquatic herb. Leaves oblong (submerged), floating leaves broadly ovate or cordate or reniform. Flowers white with yellow spots.

Fl. \& Frt.: April - November

Specimens examined: Andhra Pradesh, Srikakulam, Kaviti, Putiyadala, 27th March 2016, PV Ramana 22258 (AUV).

Pentatropis capensis (L.f.) Bullock -

APOCYNACEAE

Very slender climbing herb. Leaves ovate to ovateoblong. Flowers purplish-pink in lateral cymes. Fl. \& Frt.: July-October

Specimens examined: Andhra Pradesh, Srikakulam, Ichapuram, Burjapadu, 11 th November 2009, PV Ramana 22234 (AUV).

Pisonia aculeata L. - NYCTAGINACEAE

Shrubs or small trees with axillary spines. Leaves elliptic. Inflorescence axillary and terminal.

Fl. \& Frt.: December-February
Specimens examined: Andhra Pradesh, Srikakulam, Kaviti, 28 ${ }^{\text {th }}$ December 2010, PV Ramana 22238

(AUV).

Phyllanthus urinaria L. - EUPHORBIACEAE

Monoecious small herbs. Leaves linear-oblong. Flowers minute, greenish-white, solitary in axils. Capsule globose, verrucose.

Fl. \& Frt.: July-December

Specimens examined: Andhra Pradesh, Srikakulam, Kaviti, Putiyadala, 17th July 2016, PV Ramana 22261 (AUV).

Psilotrichum sericeum J.Konig ex Roxb.) Dalzell AMARANTHACEAE

Erect or sub-erect dichotomous herb. Leaves opposite, broadly ovate, sericeous. Flowers minute, in lax panicles.

Fl. \& Frt.: June-January

Specimens examined: Andhra Pradesh, Srikakulam, Kaviti, Ramayyaputtuga, $12^{\text {th }}$ July 2009, PV Ramana 22231 (AUV).

Rhynchosia capitata (Roth) DC. - LEGUMINOSAE

Slender trailing herb. Leaves 3-foliolate, leaflets rhomboid. Flowers yellow, in 2-4 flowered axillary raceme. Pods compressed, ovoid, hairy.

Fl. \& Frt.: October-February

Specimens examined: Andhra Pradesh, Srikakulam, Kaviti, Mutyalapeta, 28 ${ }^{\text {th }}$ October 2012, PV Ramana 22242 (AUV).

\section{Rungia repens (L) Nees - ACANTHACEAE}

Decumbent herb. Leaves elliptic. Flowers blue, in terminal spikes.

Fl. \& Frt.: September-March

Specimens examined: Andhra Pradesh, Srikakulam, Kaviti, Rajapuram, $8^{\text {th }}$ February 2009, PV Ramana 22209 (AUV).

Solanum trilobatum L. - SOLANACEAE

An armed, climbing herb. Leaves ovate-angular. Flowers violet, in extra-axillary cymes. Fruit globose.

Fl. \& Frt.: December - April

Specimens examined: Andhra Pradesh, Srikakulam, Kaviti, Pukkallapalem, 15th April 2009, PV Ramana 22211 (AUV).

Spermacoce ocymoides Burm.f. - RUBIACEAE

Erect herbs. Leaves elliptic. Flowers white, in axillary clusters.

Fl. \& Frt.: August-November

Specimens examined: Andhra Pradesh, Srikakulam, Kaviti, Rajapuram, Rajapurammetta, 28 ${ }^{\text {th }}$ August 2016, PV Ramana 22262 (AUV).

\section{Spilanthes uliginosa Sw. - ASTERACEAE}

Erect or suberect herbs. Leaves ovate. Heads yellow, elongate-ovoid, ray florets 5. Achenes dimorphic, marginal ones trigonous, central ones compressed. 
Fl. \& Frt.: November- March

Specimens examined: Andhra Pradesh, Srikakulam, Kaviti, Putiyadala Paddy fields, 21 th March 2016, PV Ramana 22257 (AUV).

Spirodela polyrrbiza (L.) Schleid - LEMNACEAE A small floating herb. Fronds herbaceous, flat above, slightly convex below. Tuft of roots from each frond.

Fl. \& Frt.: September-December

Specimens examined: Andhra Pradesh, Srikakulam, Kaviti, Putiyadala Paddy fields, 29th September, 2013, PV Ramana 22248 (AUV).

Streblus taxoides (Roth) Kurz - MORACEAE

Large shrubs with stout axillary spines. Leaves elliptic or obovate.

Specimens examined: Andhra Pradesh, Srikakulam, Kaviti, Rajapuram, Rajapurammetta, $16^{\text {th }}$

December, 2016, PV Ramana 22265 (AUV).

Synedrella nodiflora (L.) Gaertn. - ASTERACEAE Erect branched herb. Heads solitary, axillary and terminal, heterogamous, yellow, achenes dimorphic. Fl.\&Fr. Most part of the year Specimens examined: Andhra Pradesh, Srikakulam, Kaviti, Mutyalapeta, 26 ${ }^{\text {th }}$ September 2009, PV Ramana 22228 (AUV).

Trichosanthes bracteata (Lam.) Voigt -

CUCURBITACEAE

A monoecious tendril climber. Leaves palmately 3-5 deep lobed. Flowers white, axillary, male in 5-10 flowered racemes; female solitary. Fruits globose, orange red.

Fl. \& Frt.: September-November

Specimens examined: Andhra Pradesh, Srikakulam, Kaviti, Kottapalam 17th September 2015, PV

Ramana 22255 (AUV).

Wedelia chinensis (Osbeck) Merr. - ASTERACEAE Ascending herbs. Leaves oblanceolate. Heads solitary, terminal, heterogamous, yellow. Achenes of ray triquetrous, of disk compressed.

Fl. \& Frt.: June-December

Specimens examined: Andhra Pradesh, Srikakulam, Kaviti, Putiyadala paddy fields, $16^{\text {th }}$ August 2009, PV Ramana 22214 (AUV).

\section{Acknowledgements}

The author is grateful to Dr. J. Swamy, Botanical Survey of India, Deccan Regional Centre, Hyderabad, for help in identification. I am also thankful to my colleague P. Hari and to my old student P. Shanmukha Rao, for logistic support in my field trips.

\section{References}

1. Gamble, J. S. (1915-1936). "Flora of Presidency of Madras." vols. I-III. Adlard \& Son, London. (repr. ed., 2011).

2. Jain, S. K. and Rao, R. R. "A Handbook of Field and Herbarium Methods." (1977). Today and Tomorrow's Printers and Publishers, New Delhi.

3. Prayaga Murthy, P "Weed Flora of Uttarandhra." Ph.D. thesis, (2009). Andhra University. Andhra Pradesh, India.

4. Pullaiah, T. and E. Chennaiah. Flora of Andhra Pradesh." Vol. I. (1997) Scientific Publishers, Jodhpur.

5. Pullaiah, T. and A. Moulali. "Flora of Andhra Pradesh." Vol. II. (1997) Scientific Publishers, Jodhpur.

6. Pullaiah T. "Flora of Andhra Pradesh." Vol. III. (1997) Scientific Publishers, Jodhpur.

7. Rama Rao, N. and T. Ravisankar. "Additions to the Flora of Srikakulam District, Andhra Pradesh, India." Journal of Economic \& Taxonomical Botany 12.2 (1988) 483-486.

8. Rao, R.S. \& S. Harasreeramulu. "Flora of Srikakulam District, Andhra Pradesh, India". (1986). Published by Hon. Sectretary, Indian Bot. Soc. Dept. of Botany, Meerut University, Meerut.

9. Swain, P. K., Rama Rao, N. and Sanjai Mohan. "New Mangrove Habitats and Additions to the Flora of Srikakulam District, Andhra Pradesh, India". Indian Journal of Forestry. 31.13 (2008). 431-434.

\section{Cite this article as:}

Pragada Venkata Ramana. Additions to the flora of Srikakulam district, Andhra Pradesh, India. Annals of Plant Sciences 6.12 (2017) pp. 1824-1828. doi: http://dx.doi.org/10.21746/aps.2017.6.12.4 